
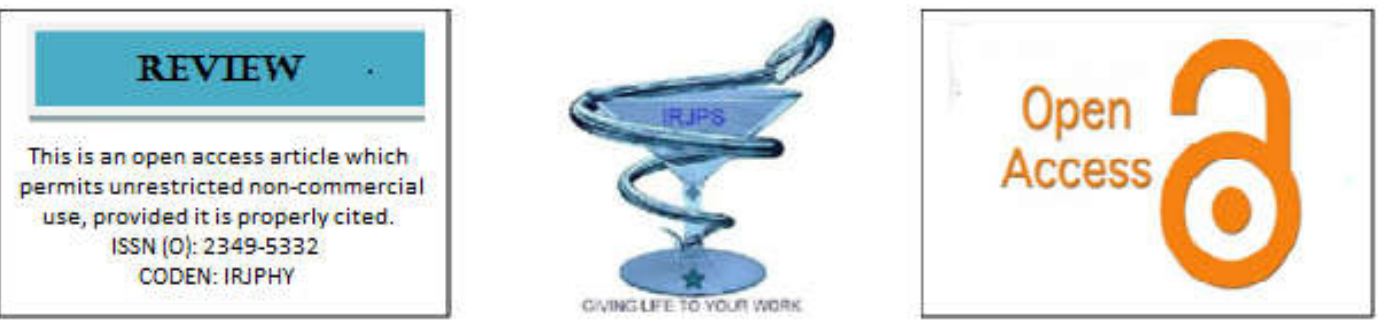

\title{
POSTPARTUM DEPRESSION
}

\section{Dr Abel Abraham Thomas*}

Department of Pharmacy Practice. Nazareth college of Pharmacy, Kerala, India

\begin{abstract}
Postpartum depression is pathological disorder characterized by non-psychotic depressive episode that begins in or prolongs into the postpartum period which usually develops between 2 and 5 weeks after delivery ${ }^{[1]}$. The patient may have hallucinations or suicidal ideation and may attempt suicide ${ }^{[2 .]}$ Untreated maternal depression can have a negative effect on child development, mother-infant bonding, and risk of anxiety or depressive symptoms in infants later in life. Management of postpartum depression is a vital part of adequate medical care. The obstetrician and paediatrician can serve important roles in screening for and treating postpartum depression [3].
\end{abstract}

Keywords : Postpartum, Depression, Pregnancy

Corresponding Author: A. A. Thomas E-mail: abelthomas119@gmail.com Mobile: +91- 8086807812
Indian Research Journal of Pharmacy and Science; 26(2020)2359-2372; Journal Home Page: https://www.irjps.in DOI: $10.21276 /$ irjps.2020.7.3.4 


\section{INTRODUCTION}

Postpartum depression (PPD) refers to a nonpsychotic depressive episode that begins in or extends into the postpartum period that usually develops between 2 and 5 weeks after delivery ${ }^{[1]}$. Like endogenous depression, it presents with a depressed mood, loss of energy, insomnia, and other symptoms. The patient may have hallucinations or suicidal ideation and may attempt suicide ${ }^{[2 .]}$ Untreated maternal depression can have a negative effect on child development, motherinfant bonding, and risk of anxiety or depressive symptoms in infants later in life. Management of postpartum depression is a vital part of adequate medical care. The obstetrician and paediatrician can serve important roles in screening for and treating postpartum depression ${ }^{[3] \text {. }}$

\section{Types of Postpartum Mood and Anxiety Disorder}

These disorders requires considerable adjustment at a highly vulnerable time for physical and emotional health, and also this is a period of risk for developing psychiatric complications such as postpartum blues, postpartum depression, and postpartum psychosis (Refer Figure 1)

\section{Postpartum Blues(baby blues)}

Of these three complications, it is the most common, yet the least severe. Postpartum blues occurs in $50-85 \%$ of women during the first few weeks postpartum. Symptoms such as irritability, anxiety, fluctuating mood, and increased emotional reactivity typically begin within one week of delivery. Treatment is not necessary since symptoms are usually mild and non-impairing, and spontaneously remit around two weeks postpartum. However, the baby blues is an early precursor of some cases of postpartum depression, particularly for women with a history of mood disorders .Therefore, women with baby blues should be monitored for increasing severity of symptoms and assessed for postpartum depression if symptoms do not remit within 12 days after childbirth.

\section{Postpartum Depression}

Postpartum depression is the second most common postpartum psychiatric complication, occurring in $13 \%$ of women. In contrast to baby blues, postpartum depression often develops insidiously three weeks after delivery and persists for at least three months. Over one-half of women remain depressed at 5-9 months, and one-third of women are still depressed from 12-18 months after childbirth. In addition to having a prolonged course, the greater severity of symptoms differentiates postpartum depression from postpartum blues.

\section{Postpartum Psychosis}

The symptoms of postpartum depression are less severe than those of postpartum psychosis, which include mixed or rapid cycling between manic and depressive episodes, agitation, delusions, hallucinations, disorganized behaviour, cognitive impairment, and low insight. However, postpartum psychosis is relatively rare, occurring in just 0.1$0.2 \%$ of women. Postpartum depression is the second most common postpartum psychiatric complication, occurring in $13 \%$ of women. In contrast to baby blues, postpartum depression often develops insidiously three weeks after delivery and persists for at least three months. Over one-half of 
women remain depressed at 5-9 months, and onethird of women are still depressed from 12-18 months after childbirth. In addition to having a prolonged course, the greater severity of symptoms differentiates postpartum depression from postpartum blues. ${ }^{[4] \text {. }}$

Figure 1: Types of post-partum depression

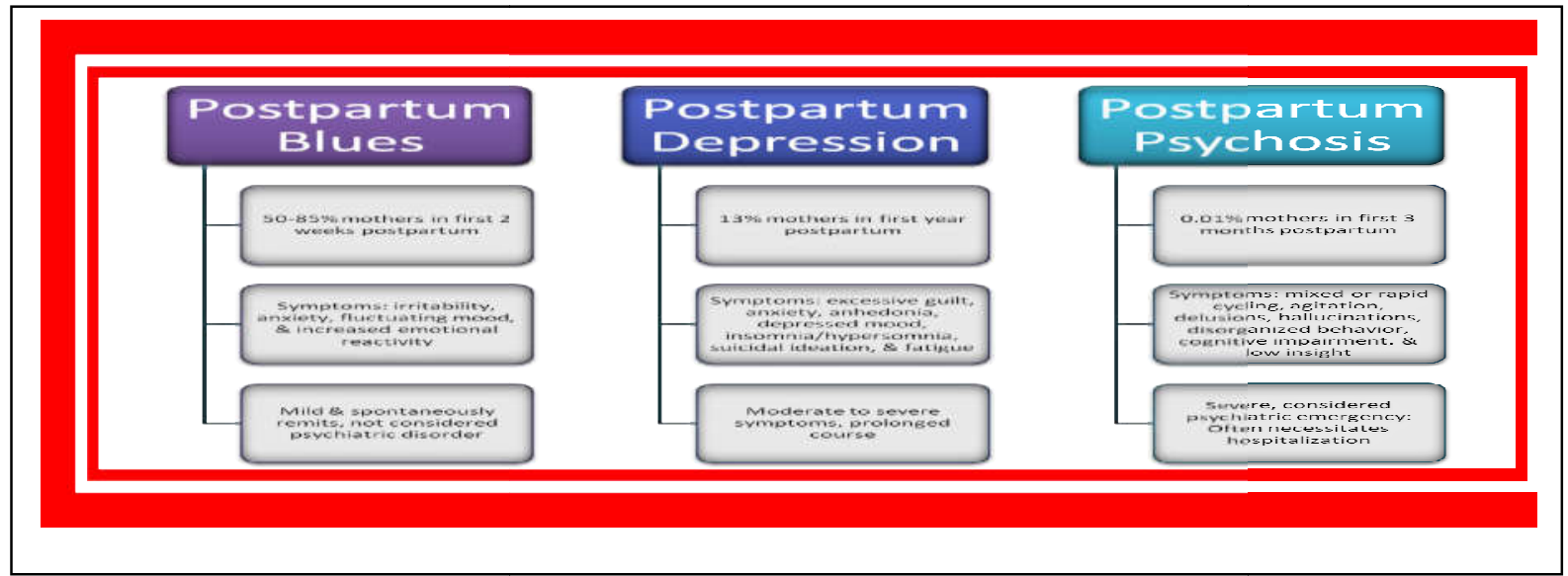

\section{RISK FACTORS}

Postpartum depression (PPD) is a mood disorder that affects 10 to $15 \%$ of new mothers. The strongest risk factor for postpartum depression is a history of postpartum major depression prior to or during pregnancy. Other important risk factors include antenatal depressive symptoms, low level of social support, major life events or stressors during pregnancy, low socioeconomic status, and obstetric complications. Untreated maternal illness disrupts the early mother-infant relationship and also contributes to short and long-term adverse child outcomes. It also has negative effects on children including increased risk of impaired mental and motor development, infant cognitive competence, poor self-regulation, and low selfesteem and behaviour problems. ${ }^{(4)}$

\section{ETIOLOGY}

It is thought that postpartum depression is caused by lack of vitamins. No specific cause of postpartum depression has been documented, but studies tend to show that more likely causes are the significant changes in a woman's hormones during pregnancy. Levels of the hormones oestrogen, progesterone, and cortical drop dramatically within 48 hours after delivery. Some women may be sensitive to hormonal changes during reproductive events, specifically menses, pregnancy, and menopause. Women who develop postpartum depression may be more sensitive to these hormonal changes and drops in hormone levels after delivery.

It includes a variety of reasons which include the following:

1. Personal history: history of depression, this can be a risk factor for postnatal depression. 
2. Birth experience: When birth experiences does not match the expectations. This feeling of being 'let down' can contribute to depression. Some women who develop postnatal depression have a traumatic or difficult birth, or a premature or unwell baby.

3 Biological factors: A small number of women who develop postnatal depression have a temporary thyroid gland defect, which is linked with mood changes. Some women may be particularly vulnerable to the drop in hormones after giving birth.

4 Changes in lifestyle: The birth of a baby can bring changes in life of a mother. She must meet the constant demands of feeding, bathing, crying and putting to sleep whereby the responsibilities of a new mother is suddenly increased. This sense of increased stress can contribute to depression.

\section{$\underline{5}$ Relationships}

The birth of a baby can also have a profound impact on the relationships with partner, family and friends. This can sometimes cause enormous strain. [10].

\section{PATHOPHYSIOLOGY}

Although there is considerable evidence for a preeminent role for three monoamine systems, serotonin (5HT), norepinephrine (NE), and dopamine (DA), in the pathogenesis of depression, depression clearly involves other circuits including neuropeptide systems such as corticotropin releasing factor (CRF) and glutamate and GABA circuits. Women are more than twice as susceptible to depression as men and in spite of the popular notion that pregnancy protects against depression, it does not.

There is increasing evidence that depression during pregnancy and also in postpartum period has adverse effects on the physiological and behavioural aspects on their off spring. (Refer Figure 2)

Figure 2: Neurobiological pattern in postpartum Depression

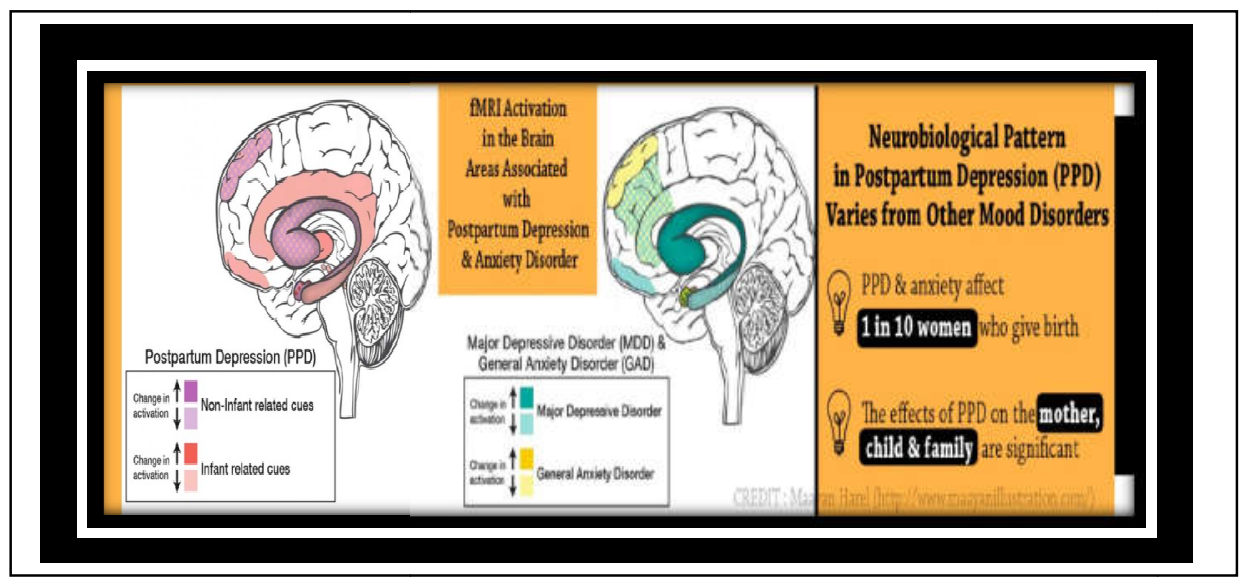

\section{DETECTION}

Criteria for a Screening Program as new screening tests arise, pressures to adopt and institutionalize screening programs emerge. However, according to diverse experts including the Joint World Health Organization (WHO)/International Agency for Research on Cancer (IARC) screening program implementation criteria; screening procedures are only justifiable if the following standards are met: 
1. Disease Issues

1.1. Conditions/diseases for which screening is used should be important health problems.

If there is an extremely low incidence of a condition, the cost and effort of screening may be prohibitive. Understanding the incidence and prevalence of the condition in a population is necessary before embarking on any large-scale screening program.

1.2. The progression of the condition should be understood; if controlled studies have demonstrated that the natural history of the condition is not favourably altered by early detection and management then screening should not be instituted.

1.3. Effective treatment for individuals with the conditions should be available

2. Screening test Issues

2.1. Screening tests should have good sensitivity, specificity, and predictive value

2.2. The screening procedure should be safe, convenient, and acceptable to the target population.

2.3. Screening tests should be cost-effective, easy to interpret, and readily incorporated Screening Test Issue

2.4. Screening tests should be accessible to the target population.

\section{Health System Issues}

3.1. A clearly defined population should be targeted.

3.2. Comparing the costs and efficiency of various screening procedures for a condition is Necessary for achieving maximum benefits at minimum cost.

3.3. An analysis of harms and benefits should be conducted. (i.e., overall long-term benefits should be greater than long-term detriment).
3.4. Strategies should be in place to ensure that the screening program will reach those who will benefit the most from the program.

3.5. Policies should stipulate what action should be taken for borderline results in order to avoid over-identifying the condition.

3.6. Standard policies for referral and preventive/treatment options that are accessible and acceptable should be established.

3.7. Facilities for screening/diagnosis and treatment should be available as the lack of follow-up negates the benefit of screening.

3.8. Responsibilities in the screening program should be clear (i.e., who does what and when).

3.9. How the findings will become part of a participant's medical record should be delineated.

3.10. Compliance with an effective care pathway should be ensured otherwise, there is no benefit of screening.

3.11. Screening programs should be an incessant process rather than being conducted once.

3.12. Continuous monitoring and evaluation should be incorporated into the screening program.

3.13. Consumer perspectives should be integrated.

3.14. Screening programs should not be static but amenable to new scientific evidence.

\section{Measures Used in the Detection of Postpartum}

\section{Depression}

Today, both general and postpartum-specific depression instruments have been utilized to measure depressive symptomatology. The validation of screening tools and the diagnosis of postpartum depression can only be accomplished through the application of diagnostic criteria such 
as the popular and progressively evolving

Diagnostic and Statistical Manual criteria for major depression in addition to the Research Diagnostic Criteria (RDC), and the International Classification of Diseases (ICD-10). Measures used to assess depressive symptomatology include standardized interviews, clinician-rated scales, and self-report questionnaires.

\section{Postpartum Depression Screening Scale (PDSS).}

It consisting of seven dimensions, each of which contains five items; the dimensions include sleeping/eating disturbances, anxiety/insecurity, emotional liability, cognitive impairment, and loss of self, guilt/shame, and contemplating harming oneself. The conceptual basis of the PDSS is based on a series of qualitative postpartum depression studies. Each item describes how a woman may be feeling after the birth of her baby and respondents are asked to indicate their degree of disagreement or agreement on a 5-point scale regarding how they have felt over the past 2 weeks ${ }^{[11]}$.

\section{Mechanism behind postpartum depression}

The biological mechanism of postpartum depression is believed to coincide with that of major depressive disorder. Depression in general is a disease of neuronal circuit integrity, which has been shown in studies by a reduction in brain volume of individuals diagnosed with major depressive disorder. Interestingly, the amount of volume loss correlates directly with the number of years of illness. Stress and depression act to reduce numerous brain proteins that promote neuronal growth and synapse formation, and antidepressant medications have been shown to increase these and other protective proteins, thereby reversing the mechanism of depression. These underlying neurobiological changes result from developmental interactions between genetic susceptibility and environmental factors (i.e., the psychosocial stresses accompanying motherhood) rather than a simple "chemical imbalance," as previously believed. Specifically, the neurobiological effects of rapid postpartum hormone withdrawal predispose women with established risk factors to postpartum depression.

An interesting distinction that makes postpartum depression unique from other depressive disorders is that it is marked by a prominent anxiety component. This may be why so many cases of postpartum depression are missed, as many Clinicians use the Patient Health Questionnaire-2which covers depressed mood and dysphoria, but not anxiety - as their primary screening technique. Indeed, $66 \%$ of depressed mothers have a comorbid anxiety disorder and should be evaluated carefully by their physicians. It is important for the physician to distinguish these feelings of anxiety as pathological and not necessarily attributed to newmother anxiety in general, so that treatment options will cover symptoms of anxiety as well as depression.

The stress of caring for a new born or even the circumstances surrounding labor and delivery may precipitate the first symptoms of postpartum depression, which has been described by nurse and postpartum depression expert Cheryl Beck as a four-stage process: encountering terror, dying of self, struggling to survive, and regaining control. Encountering terror describes the horrifying anxiety, relentless obsessive thinking, and enveloping "fogginess" that women feel as postpartum depression sets in. The dying of self is the disappearance of "normal self" that women 
experience as they go through the motions of caring for their infants, described as a "robotic" sense of "un realness." A woman struggles to survive as she attempts to improve the consequences of dying of self, seeking help from health care providers, praying for relief, or finding solace in support groups. Regaining control consists of periods of bad days interrupted by good days, until good days eventually outnumber the bad. Women may grieve during this phase for the lost time with their infants, fear recurrence, and, therefore, remain guarded about recovery. While Beck's four-stage analysis is an accurate summary of the process of postpartum depression, each woman's individual experiences should not be oversimplified.

Postpartum depression is a systemic issue affecting a woman's functioning, her sense of wellbeing, relationship with her infant and other family members, capacity for parenting, and sense of competence. As these aspects of her life become more demanding and begin to decline, the woman teeters on the brink of an emotional precipice, which has potentially grave consequences for her infant and other family members.

\section{The Effects of a Mother's POST PARTUM DEPRESSION on Her Children}

As the initial stressors related to labor, delivery, and bringing baby home give way to new triggers, infant temperament can exacerbate or minimize a new mother's postpartum depression symptoms depending on sleep patterns, frequency of crying, being easy-going or demanding, and whether or not baby is socially reinforcing with smiles and coos. 6 As the emotional toll of postpartum depression mounts in the mother with increasing guilt, a sense of being overwhelmed by child care responsibilities, and fear of being unable to cope, she may give way to bursts of uncontrollable anger, show less affection to her baby, and be less responsive to his cries. These infants in turn tend to be fussier, more distant, and make fewer positive facial expressions and vocalizations. Adverse effects on the child continue throughout the first year after birth, but postpartum depression places children of all ages at risk for impaired cognitive and emotional development as well as psychopathology. There are multiple implications for infants of mothers with postpartum depression, who's developing capacities for emotional regulation and healthy attachment relationships become compromised. These infants exhibit insecure attachments to their mothers (disorganized-disoriented), more negative, sober, flat affect, protest behaviours, regulation difficulties, and gaze aversion. They also exhibit decreased eye contact, vocalizations, activity levels, and environmental exploration. They are at risk for impaired language development and perform less well on cognitive tests at 18 months when compared to their peers of non-depressed mothers. Indeed, the effects of postpartum depression are still evident in children at ages 4-5 years old.

Female infants appear more protected against deleterious effects of postpartum depression than males. Boys with depressed mothers tend to be even more cognitively delayed than girls and display more outwardly violent behaviour. The rates of $\mathrm{ADD}$ and $\mathrm{ADHD}$ are much higher in boys than in girls. There is a correlation between boys with behavioural problems and mothers with postpartum depression. A mother's sensitivity can greatly reduce the consequences of her depression 
on the child. If she is too emotionally impaired to respond appropriately to her infant, the father (or other caregivers) can provide contingently responsive care and cognitive, emotional, and physical stimulation in order to mediate where the mother is temporarily lacking. Postpartum depression can be quickly treated and controlled. This makes it all the more crucial that it be identified as early as possible so as to reduce potentially negative outcomes, not just for the mother but for her developing infant as well ${ }^{[6] \text {. }}$

\section{Signs and symptoms}

\section{Irritability}

You may feel irritable and angry, sometimes for no reason.

\section{Anxiety}

You may feel inadequate or unable to cope. You may feel worried about things that you normally take for granted. You may not want to leave the house or meet friends. Some mothers are afraid of being left alone with their baby.

\section{Panic attacks}

You may start to have panic attacks. The symptoms include sweating hands, a thumping heart and nausea. They can happen at any time and are very distressing. You may start to avoid situations where you experience them, such as social activities, shopping, public places.

\section{Sleep problems}

You may find it hard to sleep, even when your baby is sound asleep.

\section{Tiredness}

You may feel constantly exhausted and lethargic, unable to cope with house work, looking after your baby or other tasks. You may have little interest in your appearance, in sex and in your surroundings.

\section{Concentration}

You may have trouble concentrating or feel confused or distracted.

\section{Appetite}

You may not feel like eating or you may comfort eat. As a result you may lose or put on weight.

\section{Tearfulness}

You may cry often and not always for reasons you can understand.

\section{Obsessive behaviour}

Meticulously tidying your home and trying to keep up impossibly high standards is typical of this behaviour. You may have overwhelming fears, for example about dying. Some mothers have recurring thoughts about harming their baby. Very few mothers ever act on this ${ }^{[10]}$.

\section{TREATMENT}

Treatment for postpartum depression depends on the severity of the symptoms. By definition, postpartum blues last only a few days to as much as 2 weeks. With extra help caring for the new born and emotional support for the mother, these feelings usually pass quickly. However, when depression deepens and persists for more than a short time, more active treatment is needed. If the depression remains mild enough for the woman to function, she may benefit from skilled psychotherapy. (Refer Figure 3) 
Figure 3- Treatment considerations in Post Partum Depression

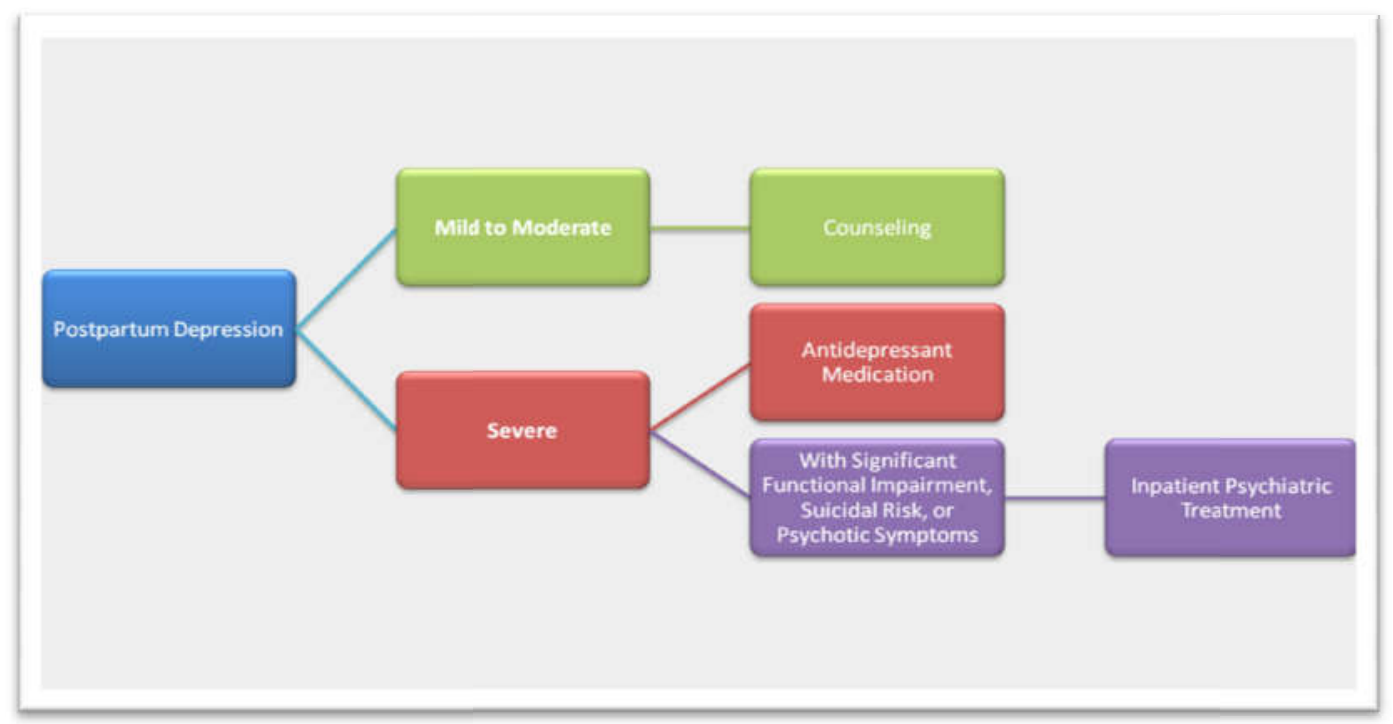

However, if there are clear symptoms of more severe major depression, experts recommend combining carefully selected antidepressant medication with counselling and support. Information has been gathered on the effects of several antidepressants on breast-fed infants, showing no evidence of serious problems. The more severe the depression, the more strongly the experts urge the use of medication. If a woman has severe symptoms, such as suicidal or psychotic thoughts, the doctor may need to put her in the hospital to ensure her safety and that of the baby while her symptoms are addressed. Electroconvulsive therapy is an alternative to consider if a mother does not respond to medication or is breast-feeding and wants to avoid medication.

\section{$\underline{\text { Antidepressant Medications }}$}

Many different kinds of antidepressants are available with different chemical actions and side effects. All of them treat depressive symptoms and may be helpful for postpartum depression. A mother who is breast-feeding, however, may be concerned about the safety of antidepressant medication for her infant. For postpartum depression in a breast-feeding mother, the experts recommend medications called serotonin reuptake inhibitors (SSRIs), which affect the brain chemical, serotonin. Their top choice among these is sertraline (Zoloft), the most widely studied antidepressant in breast-feeding mothers and their infants. While small amounts enter breast milk, little or no medication can be detected in infants, and there appear to be no adverse effects. Paroxetine (Paxil) is also a highly-rated choice. Paroxetine is not detectable in breast milk or nursing infants. Two other widely used SSRIs, fluoxetine (Prozac) and citalopram (Celexa) enter breast milk in small amounts but are viewed as acceptable alternatives. If a mother took fluoxetine or citalopram during her pregnancy and needs to stay on medication after delivery, experts do not think it is necessary to change to another drug. Tricyclic antidepressants, an older type of medication, are also viewed by experts as an

Ind Res J Pharm \& Sci|2020: Sept.: 7 (3) 2367 
appropriate choice for breast-feeding mothers. Imipramine (Tofranil) and nortriptyline (Pamelor) are 2 examples.

Tricyclics usually cause more side effects in the mother than SSRIs but are sometimes more effective. If the baby has health problems, the paediatrician can obtain a blood sample to see if the antidepressant is present in the baby in a significant amount and might be contributing to the problem. For an extremely severe type of depression in which the mother has psychotic symptoms (hallucinations or delusions), it is important to combine the antidepressant with another kind of medication called an antipsychotic. If the mother is breast-feeding, the experts recommend an older type called conventional antipsychotics (such as Haldol); newer types (atypical antipsychotics such as Risperdal or Zyprexa) are preferred otherwise, but have not been tested enough in breast-feeding mothers and their infants.

\section{Psychological treatments:}

Counselling and support for a woman with postpartum major depression, experts recommend household help and therapy with a mental health professional. If depression is severe, the experts urge finding someone to stay with and assist the mother at all times, such as a relative, friend, or paid helper. Family and friends can offer nonjudgmental support, reassurance, hope, and validation of the new mother's abilities. Common issues in psychotherapy for postpartum depression include overwhelming fears about new responsibilities and guilt over becoming depressed at such a crucial time. Two techniques that treat depression by putting these problems in perspective are interpersonal therapy andcognitive-behavioural therapy. It is usually valuable to include the spouse or other main caretaker in therapy to help him or her understand the symptoms of depression and copes with the increased stress on the family. The risk is highest in a woman who has actually had postpartum depression after an earlier pregnancy. If a woman has a history of depression, her doctor may discuss treatments to lower the chance it will return after delivery. If this is her first pregnancy and she has felt well throughout with no treatment, most experts suggest careful monitoring but no new treatment unless symptoms appear. However, if a woman has had postpartum depression in the past, most experts recommend beginning preventive treatment with antidepressant medication and psychosocial interventions right after delivery. Some experts would start a preventive program during the mother's third trimester if she is at very high risk. A typical plan might be to begin psychotherapy 2-3 months before the due date and then add antidepressant medication in the final few weeks when there is almost no risk to the foetus.

\section{Pharmacotherapy}

Support for the use of antidepressants in the treatment of postpartum depression comes from four randomized controlled trials and four open trials including sertraline, fluvoxamine, bupropion, and venlafaxine .In a double-blind study of fluoxetine versus placebo in 87 women, Appleby et al. reported that significantly more women improved in the fluoxetine group than in the placebo group. A double-blind trial comparing sertraline and nor triptyline showed no differences between medications in response rates (sertraline $56 \%$ and nortriptyline 69\%),or remission rates (sertraline $46 \%$ and nortriptyline $48 \%$ ) by the eighth week of treatment .Another study compared 
paroxetine mono therapy for 12 weeks with paroxetine plus cognitive behaviour therapy in postpartum depression with co morbid anxiety. Both groups showed significant improvement in depressive and anxiety symptoms (response rates $87.5 \%$ in the paroxetine group and $78.9 \%$ in the combination therapy group) without significant differences between groups. Because of the absence of a placebo arm in the study, it is difficult to determine the specific effect of either intervention. A study comparing paroxetine and placebo found both groups improving over time, but not differing significantly at follow-up. There was a high attrition rate, as only 31 of 70 (17 in theparoxetine group and 14 in the placebo group) participants completed the study. The response rates and remission rates in trials of treatment for moderate to severe postpartum depression ranged from $43 \%$ to $87 \%$ and $27 \%$ to $48 \%$, respectively. There is no evidence for superiority of any single antidepressant in the treatment of postpartum depression. Estrogen supplementation has also been studied in the treatment of postpartum depression, but because of the methodological flaws of these studies it is not currently recommended as a treatment option. Similarly, the mixed results on the effectiveness of omega-3 fatty acids do not support their use in the treatment of postpartum.

\section{PREVENTION OF POSTPARTUM DEPRESSION}

The recurrence rate of postpartum depression during the first year after birth has been estimated at $25 \%$ to $41 \%$, with a clustering of cases near delivery and the great majority of episodes occurring within six months after childbirth. Women with bipolar disorder, especially those who have a depressive episode after their first delivery, are at a very high risk for recurrence of postpartum depression. One study reported the recurrence rate at $100 \%$ following delivery among women who had a bipolar depressive episode after their first delivery. There have been two placebo-controlled studies of the role of antidepressants in preventing recurrent postpartum depression. In the first, a study with 22 participants, sertraline started immediately after delivery for non-depressed women with a history of at least one prior episode of postpartum depression was effective in preventing recurrence and prolonging time to relapse. However, in the second, no difference was found in the rate of recurrence in women treated after delivery with nortriptyline and those treated with placebo.

There have been no randomized controlled trials in the prophylaxis of bipolar postpartum depression. Data from retrospective studies and open trials support the use of lithium, carbamazepine, and olanzapine. A single-blind, non-randomized clinical trial comparing valproate plus symptom monitoring and monitoring without medication showed no significant differences between groups in the proportion of women who developed mania, depression, or mixed states. ${ }^{(5)}$

\section{ANTIDEPRESSANT AND BREASTFEEDING}

Some breastfeeding mothers may favour psychological interventions over pharmacological treatment. The decision for a breastfeeding mother to take medication involves a careful consideration of the potential effectiveness of drugs for postpartum depression and the potential risks of exposure of her infant to antidepressant medication. All medications pass into breast milk, but the 
extent of passage varies considerably between drugs. Citalopram, nortriptyline, sertraline, and paroxetine are first-line antidepressants because these medications in therapeutic doses are associated with low to undetectable serum concentrations in breastfed babies. Infants should be monitored for symptoms such as persistent irritability, sedation, decreased feeding, or poor weight gain. Routine laboratory testing of infant blood levels is not currently recommended because blood levels do not correlate with adverse effects. Since sleep disruption can worsen symptoms of depression, the benefits of breastfeeding need to be balanced against the reduction of the mother's opportunity for uninterrupted sleep.

\section{MAINTENANCE TREATMENT OFPOSTPARTUM DEPRESSION}

Women who are at risk for non-postpartum recurrences of depression should be evaluated for maintenance treatment. The risk factors that support the need for maintenance treatment are a history of recurrent episodes (3 or more), chronic episodes, psychotic episodes, severe episodes, episodes that are difficult to treat, significant co morbidity (psychiatric or medical), residual symptoms (lack of remission) during the current episode, and a history of recurrence during discontinuation of antidepressants ${ }^{[7]}$.

\section{DISCUSSION}

Postpartum depression is a prevalent disorder among women in the first year after birth and is considered a serious public health problem that is important to detect and treat Social support, and very young age and very low socioeconomic status may increase postpartum depression risk. A sizable group of women who experience postpartum depression are at risk for chronic depression. In addition, postpartum depression negatively affects the health of infants, children, mothers, and fathers and the overall quality of the family environment. Until screening for prenatal and postpartum depression becomes standard practice, our health care system will fail to detect the majority of women who are at risk for postpartum depression and to provide timely evaluation and effective treatment. Treatment considerations include severity of depression, whether a mother is breastfeeding, and treatment preference. Evidencebased treatments include CBT and IPT individual and group treatment, supportive counselling, psychoeducation, and antidepressant medications, specifically SSRIs. Combination approaches involving psychotherapy and psychopharmacology are recommended to treat symptoms and relational components of moderate to severe postpartum depression Biological hypothesis on the aetiology of postpartum depression are similar to those of other psychiatric disorders. However, postpartum women represent a specific group, with both hormonal and psychosocial factors that have no comparison to any other stage in a woman's life time.

There is sufficient evidence for epidemiological data and psychosocial predictors of Postpartum Depression, and relatively few studies have examined the biological aspects of this disorder. The stress model poses a plausible pathway for explaining the interaction of biological factors (eg.Variation in hormonal levels) and psychosocial factors in Postpartum Depression. The other interesting aspect of this model to explain depression is vulnerability, considering that stress in early stages (e.g. abuse, neglect, trauma) coupled 
with factors related to genetic predisposition (family history) contributes to a greater risk for depressive and anxious disorders . Concerning postpartum depression, it was shown that the presence of stressors and the personal history of depression were associated with greater vulnerability.

\section{CONCLUSION}

Postpartum depression is a common public health problem among women who have recently given birth. Given the potentially serious consequences of untreated depression for the woman and her family, it is important that health care professionals strive for early identification and treatment of postpartum depression. Because of the high rates of psychiatric co morbidity, women with depression should be screened for other psychiatric disorders such as obsessive-compulsive disorder and panic disorder. The extent literature has focused almost exclusively on unipolar postpartum depression, but a postpartum depressive episode can also occur in the patients with bipolar disorder. A depressive episode after childbirth may also herald bipolar disorder. Pregnant women with a history of postpartum depression or non-puerperal depression should be assessed regarding the need for preventive treatment of a recurrence of depression after childbirth. The available evidence suggests that antidepressants typically used to treat nonpostpartum depression are also effective in the acute, maintenance, and preventive treatment of postpartum depression. Women with mild to moderate depression may benefit from psychotherapy, including cognitive behavioural therapy, interpersonal therapy, psychodynamic psychotherapy, or non-directive counselling.

\section{REFERENCES}

1 Lanes A, Kuk L J, Tamim H. Prevalence and characteristics of Postpartum Depression symptomatology among Canadian women: a crosssectional study.BMC Public Health 2011;11:302.

2 Miyaoka Y. Postpartum depression. Journal of the Japan Medical Association 2000 ;124(1): 6567.

3 Patel M, Bailey K R, Jabeen S, Ali S. Postpartum depression: a review. Journal of Health Care for the Poor and Underserved May 2012; 23(2): 534542 .

4 Ambrosini A. Postpartum depression and melancholic type of personality: a pilot study; Journal of Psychopathology 2013; 19: 3-13.

5. https://www.journals.elsevier.com/neuralnetworks.com

6. Thurgood S, Avery M D, Williamson L.Postpartum Depression. American Journal of Clinical Medicine 2009; 6(2): 17-22.

7 Sharma V, Sharma P. Postpartum Depression: Diagnostic and Treatment Issues. J Obstet Gynaecology Can 2012; 34(5):436-442.

8 Bliszta J, Eriksen J, Buist A. Women's experience of postnatal depression - beliefs and attitudes as barriers to care. Australian Journal of Advanced Nursing; 27(3):44-54.

9 Perfetti J, Clark R, Fillmore C M. Postpartum depression: Identification, Screening, and treatment. Wisconsin Medical Journal; 103(6):5663

10 Warren P L, G McCarthy. Postnatal Depression: prevelance,mother's perspectives and treatment. Arch Psychiatr Nurs April 2007;21(2):91-100.

11 Stewart, D.E., Robertson, E., Dennis, C-L., Grace, S.L., \& Wallington, T. (2003). Postpartum 
depression: Literature review of risk factors and interventions.

12 Rychnovsky J, Beck C T.Screening for Postpartum Depression in Military Women with the Postpartum Depression Screening Scale. Mil Med .November 2006;171(11):1100-4.

13 Chen, L., Ding, L., Qi, M., Jiang, C., Mao, X. M., \& Cai, W. Z. Incidence of and socialdemographic and obstetric factors associated with postpartum depression: differences among ethnic

Han and Kazak women of Northwestern China. PeerJ, 6, e4335. doi:10.7717/peerj.4335
14 Mathisen, S. E., Glavin, K., Lien, L., \& Lagerløv, P. Prevalence and risk factors for postpartum depressive symptoms in Argentina: a cross-sectional study. International journal of women's health 2013; 5: 787-793.

15. Moraes GP, Lorenzo L, Pontes GA, Montenegro MC, Cantilino A. Screening and diagnosing postpartum depression: when and how. Trends in Psychiatry and Psychotherapy. 2017; 39(1);54-61. 